\title{
Dietary Fibre
}

Much attention and investigation is currently being directed to the importance of dietary fibre but it is difficult at this juncture to come to any firm conclusions. An interesting suggestion that has emerged from the studies on fibre is that benefit is confined to fibre coming from cereal and that the intake of fibre from fruits, vegetables, peas and beans has little relevance in the working of the protective mechanism.

In conclusion there seems no doubt that diet is one of the main contributory factors in the causation of CHD, but the impact of the message is spoiled by the absence of properly controlled acceptable scientific studies. All the evidence suggests that it is at least worthwhile to try and reduce the total intake of fat from the diet and particularly the total intake of saturated fats. These are measures, unlike other preventive measures, which cannot possibly do anybody any harm. Conversely they will almost certainly do many people a great deal of good in reducing obesity and may even help to prevent coronary heart disease.

\section{SMOKING IN THE ARMY}

\section{BRIG J P CROWDY, MB, MFCM}

Coronary heart disease is a present day major epidemic. It causes 34 per cent of all male deaths between the ages of 35 and 64. Smoking, and particularly cigarette smoking, is an important causative factor. The excess deaths among doctor smokers are 485 per $100,000,152$ of these deaths being due to coronary heart disease. For doctors aged under 45 the risk of smoking increases the prospect of mortality by 15 . Studies among civil servants have shown that the death rate from $C H D$ was increased by 64 per cent for moderate smokers and by 75 per cent for heavy smokers ( 20 or more cigarettes a day).

The effect of smoking seems to be slight where other risk factors, such as serum cholesterol, are low. The danger of smoking is accentuated as other risk factors increase. The mechanism of the action of smoking in increasing the risk from CHD is still largely unknown but it is likely that carbon monoxide both increases permeability to cholesterol and also deviates up to 15 per cent of haemoglobin with consequent loss of oxygen carriage potential. There is a possible role for nicotine which is known to increase the work of the heart and perhaps to play a role in the formation of atheromatus plaques.

\section{Smoking Habits}

National smoking habits have undergone very important changes in the last 20 years. Between 1958 and 1975 the prevalence of cigarette smoking among males of social class 1 fell from 54 per cent to 29 per cent. In the same time the prevalence among social class 5 remained virtually unchanged. In general, and for the first time since the introduction of machine made cigarettes the overall prevalence of smoking in the United Kingdom has fallen below 50 per cent (47 per cent in 1975). Smokers are thus in a significant national minority. 


\title{
Smoking in the Services
}

In 196147 per cent of newly joined Army doctors were non-smokers. Today rather more than 75 per cent are non-smokers. Unfortunately the position for soldiers is not as encouraging. In 195976 per cent of boy soldiers aged 17 years were regular smokers; by 1966 this figure had fallen to 68 per cent. But in 1975 the prevalence of cigarette smoking in the Army generally was 72 per cent, well above the national average. As an occupational group the Army is not far behind coal miners who lead the field at 79 per cent. Moreover present evidence suggests that among confirmed regular smokers, soldiers are also heavier smokers than average. The Army anti-smoking campaign has not yet met with much success, and there remains very considerable scope for positive action to reduce smoking in the Services.

\section{PHYSICAL FITNESS IN THE PREVENTION OF ISCHAEMIC HEART DISEASE}

\author{
SURG CDR D M CREAN, MB, D Phys Med, RN
}

Detailed analysis is needed to see whether exercise has a genuine role in the primary prevention of ischaemic heart disease.

Exercise is now a popular prescription, but the majority of published analyses indicate that the interpretation of the noted changes, for example, in triglyceride and catechol amine levels, in blood are subjective rather than objective. Recent work indicates that continuity of exercise programmes is more important than intermittency and it can be reasonably accepted that the physical benefit achieved will mirror the amount of training put into the programme. Furthermore, having ceased exercise it is noted that the patient returns to pre-morbid levels, with regard to itriglycerides etc quite rapidly. Speculation also exists from animal work that improvements in collateral circulation take place following exercise; the evidence that this can be extrapolated to the human heart is scanty.

From these many subjective interpretations of admittedly proven change, I can conclude that much work has to be done yet if the case is to be considered proven that physical fitness has any real role to play in the prevention of ischaemic heart disease.

\section{STRESS IN RELATION TO ISCHAEMIC HEART DISEASE}

\section{LT COL P ABRAHAM, FRCPsych, RAMC}

It can be shown that both contrived laboratory stress and events in the course of a normal working day will induce tachycardia and hypertension similar to those accompanying moderate exercise. Laboratory evidence obtained from monkeys and mice shows that both experimentally-induced conflicts within an animal, and competition between animals, can induce fixed hypertension. In addition the neuro-endocrine system has been shown to affect lipid metabolism.

However the role of stress in the development of ischaemic heart disease in 